\title{
Possibilities for a Composite Approach: Summary of the Disaster Gerontology Panel at the International College of Geriatric Psychoneuropharmacology Annual Meeting (ICGP-2014)
}

\author{
Adam Lebowitz; Athena Yi-jung Tsai, PhD, OTR; Brent P. Forester MD, MSc; \\ Hiroaki Tomita MD, PhD; Jun Shigemura, MD, PhD; and Yoshitomo Takahashi, MD
}

$\mathrm{R}$ ecent articles in this journal have considered the challenges faced by elderly survivors of disaster, such as evacuation preparedness, post-impact social isolation, and late-life combatrelated post-traumatic stress disorder (PTSD).$^{1-3}$ Last fall, a panel titled "Disaster Gerontology" was organized as part of the International College of Geriatric Psychoneuropharmacology annual meeting held in Tsukuba, Japan. Two panelists from Japan, one from Taiwan, and one from the United States presented disaster research focusing on elderly populations. Adam Lebowitz and Yoshitomo Takahashi chaired the panel. Overall, the panel highlighted the diverse approaches that must encompassed within a composite field known as "disaster gerontology."

Athena Yujing Tsai and Hiroaki Tomita discussed how trauma, loss of family and domiciles, and economic impacts can profoundly affect the health and well-being of the elderly. These populations showed health risks after Hurricane Morakot in 2009 in Taiwan and the 2011 East Japan Earthquake and tsunami. Dr. Tsai presented qualitative interview data from 18 elderly survivors and their families from 8 villages. Emptiness and distress did not appear immediately for evacuees, but started after resettlement. Dr. Tomita profiled the effects of the Tōhoku tsunami disaster from a cohort study of 2000 residents from a devastated coastal municipality in Miyagi Prefecture. After 3 years, 30\% who lost housing still exhibited post-traumatic stress responses. Effects from loss and living condition changes were prominent in the elderly, and a major factor may have been employment loss in the culturally important fishing industry. This investigation was preliminary to the Tohoku Medical Megabank Organization project, which will collect physical and mental health data from 150,000 individuals. From the data, communities are linked to appropriate resources for health promotion.

Regarding latent psychosocial sequelae from earlier traumatic events, Brent Forester presented 2 Holocaust survivor case studies. Both survivors lived full and productive lives after immigration to the United States but recently experienced depressed mood, anxiety, and cognitive impairment. Chronic stress appears to increase dementia risk, and cognitive impairment in PTSD may be an early marker for dementia. The research report of Jun Shigemura suggested other risk factors should be noted in a special population affected by the 2011 Japan disaster: workers participating in the Fukushima nuclear power facility cleanup. These workers experience anxiety from the work itself and also stress due to discrimination. These early results suggest future implications for morbidity.

The collective outcome of the panel included some important policy implications reached through taking a composite approach. Positive relationships; the ability to plan realistically, communicate, and solve problems; and the ability to manage feelings are factors that support resilience in general. The challenge is for policy to encompass initiatives promoting these factors. The importance of preserving community customs, social activity, and lifelong occupations was stressed for achieving positive mental health outcomes. Cultural sensitivities among indigenous elderly seem especially important for populations lacking the resources necessary to adapt to new environments. These mental health issues received attention in the UN Hyogo Framework for Action update in 2015 in Sendai. ${ }^{4}$ However, it was also noted that the lack of objective methods creates difficulties in evaluating post-traumatic stresses. Thus, standardization issues present in general disaster medicine are also reflected in the special population of the elderly.

\section{About the Authors}

University of Tsukuba, Graduate School of Comprehensive Health Sciences, Tsukuba, Japan (Dr Lebowitz); Department of Occupational Therapy, College of Health Sciences, Kaohsiung Medical University, Kaohsiung, Taiwan (Dr Tsai); Geriatric Mood Disorders Research Program, McLean Hospital-Harvard Medical School, Belmont, Massachusetts (Dr Forester); Department of Disaster Psychiatry, International Research Institute of Disaster Science, Tohoku University, Sendai, Japan (Dr Tomita); Department of Psychiatry, National Defense Medical College, Tokorozawa, Japan (Dr Shigemura); and Department of Disaster Psychiatry, School of Medicine, University of Tsukuba, Tsukuba, Japan (Dr Takahashi). 
Correspondence and reprint requests to Adam Lebowitz, PhD, University of Tsukuba, Graduate School of Comprehensive Health Sciences (ajlebo@gmail.com).

Published online: July 9, 2015.

\section{REFERENCES}

1. Roush RE, Tyson SK. Geriatric emergency preparedness and response. Disaster Med Public Health Prep. 2012;6(4):385-392.
2. Gibson M, Gutman G. Mitigating the risk of late-life mental health problems for veterans: contributions of the life course perspective to emergency management. Disaster Med Public Health Prep. 2013;7(3): 332-335. doi:10.1001/dmp.2010.48

3. Inoue M, Matsumoto S, Yamaoka K, et al. Risk of Social Isolation Among Great East Japan. Disaster Med Public Health Prep. 2014;8(4):333-340. doi:10.1017/dmp.2014.59

4. Tomita H, Ursano RJ. Breakout session 3 summary: psychosocial/mental health concerns and building community resilience. Disaster Med Public Health Prep. 2014;8(4):363-365. doi:10.1017/dmp.2014.68 